\title{
COMPleXity Perspective ANd ENGINEERING SySTEMS - NOVEL APPROACHES
}

\author{
Sladjana Gajic ${ }^{\mathrm{a}}$, Iztok Palcic ${ }^{\mathrm{b}}$, Ilija Cosic ${ }^{\mathrm{a}}$ \\ ${ }^{a}$ University of Novi Sad, Faculty of Novi Sad, Trg Dositeja Obradovica 6, Novi Sad 21000, Serbia \\ ${ }^{b}$ University of Maribor, Faculty of Mechanical Engineering, Smetanova ulica 17, 2000 Maribor, Slovenia
}

\begin{abstract}
Systems perspective is nowadays expanded to complexity perspective, by including aspects of disorder, instability, nonlinearity, unpredictability, and it is widely applied to different disciplines. The purpose of this paper is to contribute to engineering systems theory by observing projects as complex adaptive systems (CAS). Complexity theory could give some insights in the way organizations interact, relate, and evolve within their environment. Authors of this paper used literature review as a methodological approach, and focused on structural and dynamic complexity of systems. There is a need for understanding complex systems with numerous techniques and algorithms necessary for an engineer to operate in complex and dynamic environments (e.g. production and manufacturing). Implications of this paper could be used as a basis for further research that copes with complexity of CAS, as well as in bridging complexity concepts and managerial reality.
\end{abstract}

Keywords: compexity; CAS; project management; engineering systems
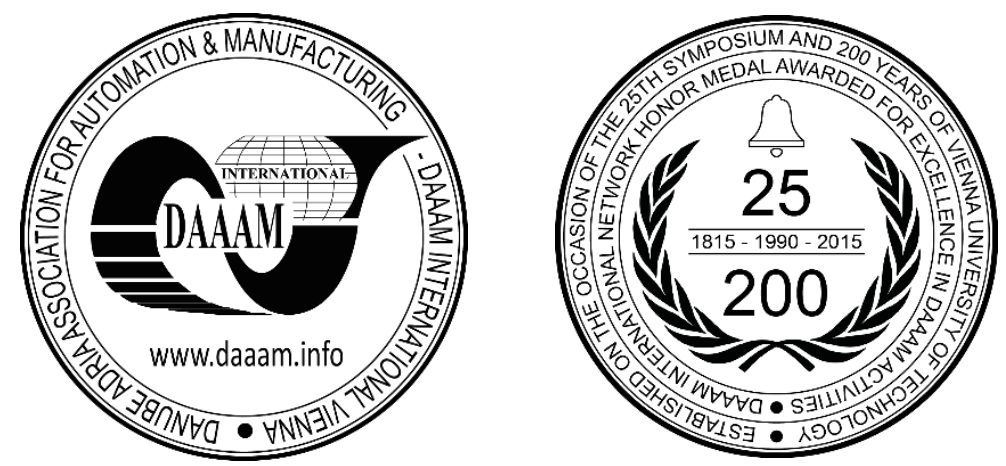

This Publication has to be referred as: Gajic, S[ladjana]; Palcic, I[ztok] \& Cosic, I[lija] (2016). Complexity Perspective and Engineering Systems - Novel Approaches, Proceedings of the 26th DAAAM International Symposium, pp.10911096, B. Katalinic (Ed.), Published by DAAAM International, ISBN 978-3-902734-07-5, ISSN 1726-9679, Vienna, Austria

DOI: 10.2507/26th.daaam.proceedings. 153 


\section{Introduction}

In today's dynamic business environment characterized by uncertainty and continuous change, there is a need for new approaches that can tackle the complexity of systems [1]. Traditional approaches that observed systems as relatively predictable, controlled, goal oriented and centralised, are no longer appropriate. The world has moved radically towards greater complexity in the last two centuries [2].

The purpose of this paper is to theoretically analyse complexity of engineering systems and its relationship with complex projects, and to propose potential ideas for the future research. There is a need for further research in this field, for academics, who are studying this topic, as well as practitioners, that have to deal with project complexities on a regular basis. Complexity theory evolved from different disciplines: mathematics, physics, biology, life science, economics, organizational science, and computational intelligence in the middle of twentieth century, and later offered its insights to other disciplines [3][4][5]. Implications of this paradigm led to new approaches, which now have to handle complexity in dynamic systems, abandon traditional cause and effect models, and apply nonlinear approaches.

This paper is organised as follows: the second chapter covers the most important concepts of complexity theory - Complex Adaptive Systems, butterfly effect, implications of complexity theory to organisational theory and engineering systems perspective to complexity management of projects. Following this, authors covered possible strategies for managing project complexity, gave suggestions for further research and finished with final conclusions.

\section{Literature review}

\subsection{Complexity theory}

Complexity theory is very much concerned with the study of the structure and dynamics of complex adaptive systems (CAS), characterised with nonlinearity, self-organization, and emergent properties [6]. Organisations and projects with their characteristics can be observed as complex systems.

Nonlinearity is the term closely related to chaos theory. In this context, a small error at the beginning of the project can lead to huge error later in the process [7]. Edward Lorenz [8] was the meteorologist who was best known for introducing the term "butterfly effect". Attractors are behaviors of nonlinear complex systems, and the most famous attractor is the Lorenz's strange attractor, shown in the Figure 1. The shape of the strange attractor represents specific path of behavior in chaos, a hidden boundary that implies that the system does not wander off into infinity, but that the boundary lives within the system. Although systems' behavior is unpredictable, it does have a hidden pattern, a qualitative shape (Figure 1) [1]. Lorenz claimed that linear changes are no longer possible, since a small change in a system's initial state, can lead to significant change when observed in long terms. Systems are constituted of the small parts that have surprising effects on the whole, and changes that occur at the system level might be the result of the interactions that have happened at the lower levels [9] [10].

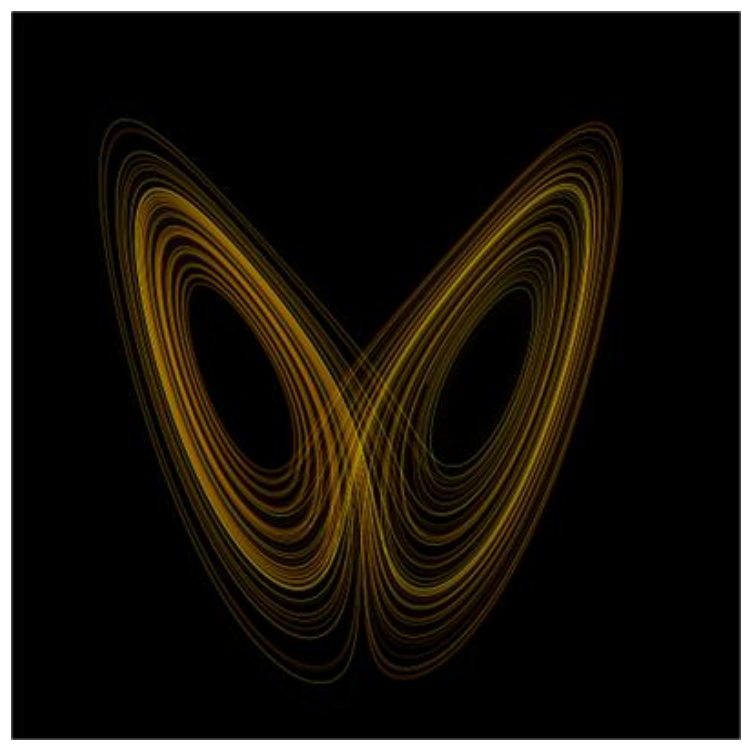

Fig. 1. Lorenz's strange attractor [8]

Complexity theory is widely applied to different disciplines, and its purpose is to help in understanding dynamics of complex human and social and natural systems, observed over time [11]. Complexity theorists holistically observe the system, without making conclusions about systems based on linearity and predictability. Most important concepts of complexity science, and their evolution can be summarised in the Table 1 [1]. 


\begin{tabular}{llll}
\hline Period & Concepts & Key researcher & Discipline \\
\hline $1960 \mathrm{~s}$ and & Butterfly effect & Edward Lorenz & Life Science \\
$1970 \mathrm{~s}$ & Strange attractors & Edward Lorenz & Mathematics \\
& Self-organizations, & David Ruelle & \\
& Ilya Prigogine & Physical Science, \\
& Sissipative structures & Stuart Kauffman & Life Science \\
& Evelf-organizations, & & \\
& Evolution and complexity & & \\
\hline $1980 \mathrm{~s}$ & Edge of chaos & Chris Langton & Life Science \\
\hline onwars and & CAS & John Holland & Mathematics \\
& Emergence & Murray Gell-Mann & Physical Science \\
& Complex responsive process of & Chris Langton & Life Science \\
& relating & Ralph Stacey & Life Science \\
\hline
\end{tabular}

Table 1. Complexity science evolution [1]

There are different types of complexity, but in this paper authors were focused on structural and dynamic complexity. Analysing only structural complexity (breaking down systems to the smaller constitute parts) can lead to wrong conclusions about system, especially about its change over time.

Minai claims that "complex systems engineering is not primarily focused on producing predictable, stable behaviour within carefully constrained situations, but rather to systems capable of adaptation, change and novelty" [12]. Braha states that Complex Engineered Systems (CES) have emerged as a result of new technologies, such as the Internet, GPS, wireless networking, micro-robotics and nanotechnology [2]. These engineered systems now have to manage different issues: adaptability, self-organization, resilience, robustness, durability, reliability, self-monitoring, and selfrepair, that appeared as a result of evolution. They produce the need for more effective ways of their management.

Complexity scientists especially focus on Complex Adaptive Systems (CAS), due to their specifics such as nonlinear behaviour, emergent properties, and possibility to self-organise. Stacey [13] defined CAS as systems consisted of "a number of components, or agents, that interact with each other according to sets of rules that require them to examine and respond to each other's behaviour, in order to improve their own behaviour". Following this, projects could be observed as Complex Adaptive Systems, with numerous activities, complex web of stakeholders and limitations in time and scope.

CAS's models offered a holistic way for complex systems analysis, without abstracting their interdependencies and nonlinear interactions. They typically show how complex outcomes depend on interconnection between agents [14]. This is particularly important for organizational scholars because interdependency is important for analysis of modern organizations [15].

\subsection{Importance of complexity theory for organisational theory}

Langton [16] defined "the edge of chaos" as a condition where stability and instability occur simultaneously. If an organization wants to survive on a dynamic market, it has to innovate. Complex systems must operate in far from equilibrium condition since nothing novel can emerge in systems with high degrees of stability.

This paper will include Turner and Müller's interpretation of projects as temporary organisations, created to generate changes [17]. Projects are considered to be one of the basic means for achieving competitive advantage and success in business operations [18] [19]. [20]. In order to adapt to continuous changes in the environment, organisations have to be flexible and able to offer quality, variety, novelty and functionality of its products, and they are able to perform this through projects [21]. Projects, with their specific characteristics, are observed as CAS in this paper.

Cause and effect models and traditional linear thinking, characteristic for traditional analysis of systems, are no longer appropriate methods. CAS require new nonlinear framework that acknowledges that linear approaches are inherently over simplistic.

One of the most important concepts of complex systems is self-organisation, which can be described as the spontaneous appearance of organization through limited interactions among simple components. Examples of selforganizing systems are visible in the nature (e.g. galaxies, tornadoes, cells), and almost none of the systems engineered by humans is able for self-organisation. Complexity theorists aim to make complex systems similar to systems in the nature, by stimulating their self-organisation [2].

Pascale et al. [22] proposed a management model derived from complexity theory, based on four core principles:

1. Equilibrium is a precursor to death. When a system is in a state of stability, it is not highly responsive to changes and is risking its survival.

2. When faced with either an opportunity or a threat, systems move toward the edge of chaos in which solutions to the challenges are more likely to be discovered.

3. When doing this, they go through self-organizing process and new forms and behaviours emerge from the upheaval. 
4. Unpredictable consequences are inevitable. The challenge for organisations is to move these systems in a way that is similar to the desired outcomes.

Classical engineering systems are characterized with predictability, reliability, stability, precision and controllability, and aim to produce efficient systems that are reliable and meet pre-specified constraints and pre-specified standards of performance in pre-specified situations. Traditional engineering usually gives single solution for the specified problem, and tries to explain system by applying only structural approach to understanding complexity.

When applied to complex context, traditional approaches show numerous drawbacks. In addition, natural complex systems prove to be much more efficient in problems solving. Yum [23] highlights that complex systems engineering does not aim to produce predictable, stable behaviour within carefully constrained situations, but to obtain systems capable to adapt, change and produce novelty and even surprise.

Projects could be observed as complex, self-organising systems, with their specifications (requirements and constraints) that undergo design process that is highly social, consisting of hundreds of designers, customers, and other participants. These actors reshape the requirements and potential solutions through continual negotiations, evaluations, and revision, and increase their complexity [2].

\section{Complexity management of projects in engineering systems}

Modern world is governed by different engineering systems that fulfil central societal functions with sociotechnological systems. They are development with help of projects, programmes and portfolios [24]. Projects are heart of organisations in different industries, from construction, software development, systems engineering, and product development [25]. This paper covers two aspects of complexity that tackles projects [26]:

1) Structural complexity - number and types of elements and their relationships in the system (static snapshot of a system in particular point of the time);

2) Dynamic complexity - behaviour of a complex system (elements and their relationships and the structure of the system). This tackles duration of the project, changes in staffing, organisational resistance to changes etc.

Complexity in engineering projects is determined by the technical complexity of the system that is being developed, and organisational complexity of the system that is developing the product or a service [24].

PMIs White Paper [19] proposed possible strategies for dealing with complexities in projects. When it comes to structural complexity, authors suggested Network analysis, Black-Boxing and Modularity.

Network analysis shows the elements of the system, and binary indicate existence or absence of relationships between these elements [24]. Network analysis approaches differ mostly in their graphical representation of the elements; the most well known types are matrix-based and graph-based network analyses. Some of the examples are organisational charts, social network analysis, IDEF0 diagrams, workflow diagrams, and PERT diagrams [27].

Network analysis could also be based on modelling data, such as modelling flow of the information between processes or the dependence between system's technical components.

Another type of complexity is dynamic complexity. Dynamic Complexity can be analysed trough System dynamics, aimed at overcoming key social challenges of complex systems, such as bounded rationality and thinking and decision-making biases [24].

Wicked problems are the type of complex project environment that occur when true requirements of the customer are unknown before project starts, and they develop in parallel with the solution [24]. Treating wicked problems as simple could lead to chaos in projects.

Roberts [28] suggested three strategies for managing wicked problems faced by complex projects: authoritative, competitive and collaborative. Table 2 offers description of each of the strategies (Table 2).

\begin{tabular}{|c|c|c|}
\hline & Main idea & Description \\
\hline Authoritative & $\begin{array}{l}\text { Reducing stakeholder } \\
\text { complexity }\end{array}$ & $\begin{array}{l}\text { Problems are simplified; } \\
\text { Number of stakeholders is decreased to few people; } \\
\text { Disadvantage: authorities and experts charged for this may } \\
\text { not encounter all the perspectives of the problem; }\end{array}$ \\
\hline Competitive & $\begin{array}{l}\text { Developing alternative } \\
\text { system models }\end{array}$ & $\begin{array}{l}\text { Pitting opposing points of view against each other; } \\
\text { Advantage: assessment of potential solutions; } \\
\text { Disadvantage: confrontational environment where } \\
\text { knowledge sharing is discouraged; significant resources are } \\
\text { required; }\end{array}$ \\
\hline Collaborative & Extensive system modelling & $\begin{array}{l}\text { Engaging all of the stakeholders in order to find the best } \\
\text { possible solution for all of them; }\end{array}$ \\
\hline
\end{tabular}

Table 2. Strategies for managing wicked problems [28] 
Through this complex view, projects, as important parts of engineering systems, could be seen as complex adaptive systems. Authors of this paper presume that complexity of projects has important influence on their management style and overall success.

Oehmen claims that there are three types of complex projects [24]:

1. Simple - requirements are known, waterfall implementation;

2. Complex - requirements are changing throughout project life cycle; feedback loops and unforeseen emergent behavior, but are fundamentally still manageable by structured analysis - e.g. agile projects;

3. "Chaotic" - change faster than we can observe and learn, analytic techniques are not applicable, and management is relied on robust decision-making heuristics.

In this chapter, authors gave the summery of possible strategies for analyzing complexity and treating possible problems that occur during complex project implementation. Further research could benefit from testing these implications in real systems.

\section{Implications of the study}

Implications of complexity theory are significant for the organisational theory due to their insights about interaction, relation, and evolution of organisations within their environments, as well as analysis of their structure, relationships between elements and behaviour over time. Complex systems engineering aim at producing systems capable of adaptation, change and novelty, and projects can be seen as a possible tool for delivering these changes. In this sense, correlation between dynamic complexity and possible agile approaches for managing projects seems to be of interest in the future research.

Dynamic complexity follows the behaviour of a system over time. One of the possible problems that increase dynamic complexity are so-called "wicked problems" that occur when initial requirements of the customer are unknown or changed during the project life cycle. If complexities of projects are ignored, and managing styles are not adjusted, they can lead to chaotic behaviour of the system. Authors of this paper aim to show in wider research whether overall project success is correlated with managing complexities of projects, flexibility of managing style and project environment.

Limitation of the study could be a small number of evidences or justifications that projects are complex systems, so this is an important topic for further research. Applying different methodologies for analyzing project complexity, and suggesting possible models for treating complexity in project environments would also be interesting for future researchers.

\section{Conclusion}

Complexity theory provides novel perspective to understanding how organisations operate in complex and uncertain environments. Authors of this paper attempted to provide comprehensive picture of the complexity of projects, by relating it to wider concept of systems' complexity. This paper has systematically reviewed the literature on the complexity of engineering systems, investigated the relevance of complexity theory to project analysis and gave possible strategies for dealing with complexity on projects. PMIs White Paper [19] deals with engineering systems perspective to complexity management to projects. Research in our paper adds perspective of Complex Adaptive Systems, and proposes possible practical testing of this dimension in future research. Results of first authors' future doctoral research would show how overall project success is correlated with complexity of projects, flexibility, planning process, methodology of implementation, inclusion of beneficiaries in all PLC phases, and project environment.

The further research could benefit from implementing strategies for dealing with complexity to managing real systems, and linking them with principles of flexibility and adaptability. Assessment of the complexities could result in the indicators that can be monitored and managed.

Relationship and impact between structural and dynamic complexity is also one of the interesting research fields in the future. More studies that provide necessary evidences or justifications that projects are complex systems are desirable in the future academic papers.

\section{Acknowledgements}

This article has been produced as part of a research project "Development of software for managing overhaul and installation of rail vehicles braking systems" under Grant number 035050, partially supported by the Ministry of Education, Science and Technology Development of the Republic Serbia. 


\section{References}

[1] Z. Ying and L. S. Pheng, Project Communication Management in Complex Environments, 2014.

[2] D. Braha, N. Suh, S. Eppinger, M. Caramanis, and D. Frey, Complex engineered systems. Springer, 2006.

[3] J. H. Holland, Emergence: From chaos to order. Oxford University Press, 2000.

[4] S. A. Kauffman, "At home in the universe: the search for laws of self organization and complexity," ISBN-13, pp. 978-195111309, 1996.

[5] R. D. Stacey, Strategic management and organisational dynamics: The challenge of complexity to ways of thinking about organisations. Pearson education, 2007.

[6] E. McMillan, Complexity, organizations and change. Routledge, 2004.

[7] J. Crutchfield, J. Farmer, N. Packard, and R. Shaw, "Chaos," Sci. Am., 1986.

[8] P. R. Lawrence, J. W. Lorsch, and J. S. Garrison, Organization and environment: Managing differentiation and integration. Division of Research, Graduate School of Business Administration, Harvard University Boston, MA, 1967.

[9] S. Kelly and M. A. Allison, "The complexity advantage," How the Science of Complexity Can Help Your Business Achieve Peak Performance. Achieve Peak Performance. New York, 1999.

[10] T. Carroll and R. M. Burton, "Organizations and complexity: Searching for the edge of chaos," Computational \& Mathematical Organization Theory, vol. 6, no. 4, pp. 319-337, 2000.

[11] M. C. Jackson, Systems approaches to management. Springer Science \& Business Media, 2000.

[12] Y. Bar-Yam,"Large scale engineering and evolutionary change: Useful concepts for implementation of forcenet." Report to Chief of Naval Operations Strategic Studies Group (2002).2002.

[13] R. D. Stacey, Complexity and creativity in organizations. Berrett-Koehler Publishers, 1996.

[14] P. Anderson, "Perspective: Complexity theory and organization science," Organization science, vol. 10, no. 3, pp. 216-232, 1999.

[15] J. D. Thompson, Organizations in action: Social science bases of administrative theory, vol. 1. Transaction publishers, 2011.

[16] C. G. Langton, "Artificial Life, Santa Fe Institute Studies in the Science of Complexity," in Proceedings of an Interdisciplinary Workshop on the Synthesis and Simulation of Living Systems, 1988.

[17] J. R. Turner and R. Müller, "On the nature of the project as a temporary organization," International Journal of Project Management, vol. 21, no. 1, pp. 1-8, 2003.

[18] Z. Kremljak, I. Palcic, and C. Kafol, "Project Evaluation Using Cost-Time Investment Simulation,” International Journal of Simulation Modelling (IJSIMM), vol. 13, no. 4, 2014.

[19] G. D. Wu, "Project-Based Supply Chain Cooperative Incentive Based on Reciprocity Preference," International Journal of Simulation Modelling (IJSIMM), vol. 13, no. 1, pp. 102-115, 2014.

[20] D. Dougherty, "Interpretive barriers to successful product innovation in large firms," Organ. Sci., vol. 3, no. 2, pp. 179-202, 1992.

[21] J. R. Turner, The handbook of project-based management, vol. 92. McGraw-hill, 2014.

[22] R. T. Pascale, M. Milleman, and L. Gioja, "Surfing the edge of chaos," Sloan management review, vol. 40, no. 3, 1999.

[23] Y. Bar-Yam, “General features of complex systems,” Encycl. Life Support Syst. (EOLSS), UNESCO, EOLSS Publ. Oxford, UK, 2002.

[24] J. Oehmen, C. Thuesen, P. P. Ruiz, and J. Geraldi, "Complexity Management for Projects, Programmes, and Portfolios: An Engineering Systems Perspective,” Project Management Institute, PMI, 2015.

[25] H. Rahmandad, K. Hu, and T. Ratnarajah, "Modeling rework cycle: comparing alternative formulations," System Dynamics Review, vol. 26, no. 4, pp. 291-315, 2008.

[26] H. R. Maylor, N. W. Turner, and R. Murray-Webster, "How hard can it be? Actively managing complexity in technology projects," Research-Technology Management, vol. 56, no. 4, pp. 45-51, 2013.

[27] T. R. Browning and R. V Ramasesh, "A survey of activity network-based process models for managing product development projects," Production and Operations Management, vol. 16, no. 2, p. 217, 2007.

[28] N. Roberts, "Wicked problems and network approaches to resolution," International public management review, vol. 1, no. 1, pp. 1-19, 2000. 